\title{
Prevalence of chronic kidney disease among HIV- infected adults on antiretroviral therapy in northern Namibia: a cross-sectional study
}

\author{
Michael Mboko, Tonya Marianne Esterhuizen, Mogamat Razeen Davids \\ Division of Epidemiology and Biostatistics, Stellenbosch University, Cape Town, South Africa; Division of Nephrology, Stellenbosch \\ University and Tygerberg Hospital, Cape Town, South Africa.
}

\section{ABSTRACT}

Introduction: There is an epidemic of chronic kidney disease (CKD) in Africa and human immunodeficiency virus $(\mathrm{HIV})$ infection is among the major drivers. However, the burden of CKD in HIV-infected patients in Africa varies widely by country and study, ranging from 0.5-59.3\%. Published data on the prevalence of CKD in the Namibian HIV-infected population are scarce. In this study, we aimed to estimate the prevalence of CKD and associated factors in HIV-infected adults on antiretroviral therapy in northern Namibia.

Methods: We conducted a cross-sectional study in the four regions of northern Namibia, using existing electronic records used in the management of HIV-infected patients. Variables captured included the two most recent serum creatinine measurements, date of birth, sex, date of initiating antiretroviral therapy, current antiretroviral treatment, and most recent HIV viral load results. We used standardised serum creatinine measurements to estimate the glomerular filtration rate (GFR) using the Chronic Kidney Disease Epidemiology Collaboration (CKD-EPI) equation. CKD was defined as estimated GFR (eGFR) $<60 \mathrm{~mL} / \mathrm{min} / 1.73 \mathrm{~m}^{2}$ on two occasions at least three months apart. Factors associated with CKD were assessed using logistic regression.

Results: We included I 993 participants, of whom | 362 (68\%) were female and mean age was $44.5 \pm$ II.5 years. The proportion of participants who were virally suppressed was $97 \%$ (95\% Cl 96, 98\%) and the median duration on antiretroviral therapy was 107 months (IQR 62-149). The prevalence of CKD was I.4\% (95\% Cl I.0, 2.0\%). CKD cases were 13 times more likely to be 45 years or older and 3.5 times more likely to be male than those without CKD.

Conclusions: Our findings suggest a low prevalence of CKD among the HIV-infected population in northern Namibia. Patients older than 45 years may need additional monitoring for kidney function using eGFR.

Keywords: chronic kidney disease; HIV; eGFR; Namibia.

\section{INTRODUCTION}

Chronic kidney disease (CKD) is a condition characterized by abnormalities of kidney function or kidney structure, that have been present for at least 3 months, with implications for health $[\mathrm{I}]$. The consequences of CKD include the development of kidney failure and a 30-fold increase in cardiovascular disease mortality in patients with CKD compared to the general population [2]. Symptoms of CKD are non-specific or absent until the disease is advanced [3]. This makes screening essential for earlier diagnosis and intervention to improve outcomes. The diagnosis and classification of CKD is based on measurement of markers of kidney damage such as persistent proteinuria, estimation of the glomerular filtration rate (GFR), and radiological imaging for abnormal kidney structure $[3,4]$.

Prediction equations are used to estimate the GFR from measurements of serum creatinine and current guidelines 
recommend the use of the Chronic Kidney Disease Epidemiology Collaboration (CKD-EPI) equation [I]. Methods used to estimate GFR have an important influence on the diagnosis of CKD, the stage of CKD, and the reported burden of CKD [4]. Variation in creatinine assays may lead to interlaboratory variations in serum creatinine measurements and, in turn, estimated GFR (eGFR). Hence, measurement and reporting of standardized serum creatinine is recommended, where the assay is calibrated to isotope dilution mass spectrometry (IDMS)-traceable reference materials. An eGFR of less than $60 \mathrm{~mL} / \mathrm{min} / 1.73 \mathrm{~m}^{2}$ for at least 3 months is used as the threshold to diagnose CKD [I].

There is an epidemic of CKD in Africa that is being driven by diseases such as diabetes mellitus and hypertension, human immunodeficiency virus (HIV) infection, pregnancy complications, use of herbal or traditional medicines and trauma [4,5]. Diabetic nephropathy is a common complication of diabetes mellitus that may result in CKD, and its occurrence is increasing in Africa [6]. Hypertension is reported to be an important cause of CKD, though it can occur secondary to pre-existing kidney disease [7]. SubSaharan Africa is the region most affected by HIV infection, with 26 million people living with HIV. Highly active antiretroviral therapy has greatly improved the survival of HIVinfected people [8]. This improved survival has resulted in HIV infection occurring in the presence of noncommunicable diseases, leading to an elevated risk of CKD among HIV-infected patients [8,9]. Further-more, infection with HIV and treatment with certain antiretroviral medicines has been associated with kidney pathology such as glomerular damage, tubular injury, and chronic inflammation [10]. HIV-infected patients develop CKD due to HIV-associated nephropathy (HIVAN), HIV-related immune complex disease, chronic inflammation, or subsequent to acute kidney injury (AKI) associated with opportunistic infections or medicine toxicity $[2,10]$. Tenofovir disoproxil fumarate (TDF) is a reverse transcriptase inhibitor used in the treatment of HIV infection. TDF-related kidney injury is more likely in patients with pre-existing kidney impairment, advanced HIV disease, diabetes mellitus, hypertension, hepatitis B coinfection and concurrent use of nephrotoxic medicines $[4,10]$. Genetic susceptibility due to polymorphisms in the apolipoprotein LI (APOLI) gene puts individuals of African descent particularly at risk of CKD $[2,10]$.

CKD in the HIV-infected population leads to poor oucomes, including an increase in mortality $[\mathrm{I} I$ ]. In a study conducted in South Africa, kidney disease was an important risk factor for increased in-hospital mortality among HIV-infected patients [12]. Additionally, inadequate monitoring of kidney function was recognized as a modifiable risk factor contributing to elevated mortality in HIV-infected adults in Namibia [ 13]. If CKD progresses to kidney failure, lifesaving kidney replacement therapy (KRT) or kidney transplantation will be required. However, there are marked disparities in access to KRT, especially among African countries. The burden of HIV-associated kidney disease varies widely by country in Africa, and ranges from $0.5 \%$ to $59.3 \%$ $[5, \mid 4-16]$. Part of this variation is thought to be a result of varying methods used to assess kidney function, differences in the definition of kidney disease and in the equations used to estimate the GFR [5]. Many studies use only a single eGFR estimate to assess CKD prevalence, leading to overestimation of the burden of disease [14]. There are concerns that an epidemic of HIV-associated CKD may go unrecognized due to the lack of good quality data from sub-Saharan Africa [8]. As such, reviews conducted on CKD prevalence in Africa have concluded that there is a need to generate good quality prevalence data on CKD in HIV from sub-Saharan Africa [5, I4, I5].

Namibia is a country in southern Africa with an estimated population of 2.5 million people, divided into 14 regions, and is classified as an upper-middle-income country by the World Bank. The country has a high burden of HIV infection, with an estimated prevalence of $12.6 \%$ among those aged from 15 to 64 years [ 17]. HIV prevalence is highest in the Zambezi region at $22.3 \%$, followed by the four regions of northern Namibia, ranging from 15.8\% to 17.9\% [17]. Guidelines for the management of HIV infection have been produced, which recommend laboratory monitoring of kidney function as part of the standard care [18]. The country's guidelines recommend the use of creatinine clearance as an indicator of kidney function and it is estimated based on serum creatinine measurements using the Cockcroft-Gault equation [18]. Kidney function is assessed at baseline, 6 weeks from baseline, 6 and 12 months from baseline, and annually thereafter [18]. However, routine monitoring for persistent proteinuria is not performed. Despite the high burden of HIV infection, there is a lack of published data on the prevalence of CKD among $\mathrm{HIV}$-infected adults in Namibia. Considering the potential disease burden, the high cost of treatment for kidney failure, and disparities in the availability of KRT, it is important to determine the prevalence of CKD associated with HIV infection and the related risk factors.

The main objective of the study was to estimate the proportion of patients with CKD among HIV-infected adults on antiretroviral therapy. The secondary objective was to assess the demographic and clinical factors associated with CKD in this population. 


\section{METHODS}

We carried out a cross-sectional study where data were obtained from the electronic database used in the management of HIV-infected patients in the public healthcare sector in Namibia. A retrospective record review of electronically available datasets was carried out at hospitals in the Ohangwena, Omusati, Oshana and Oshikoto regions of northern Namibia. Participating hospitals were Eenhana and Engela District Hospitals in Ohangwena region, Oshikuku and Outapi District Hospitals in Omusati region, Oshakati Intermediate Hospital in Oshana region, and Onandjokwe Intermediate Hospital in Oshikoto region. The antiretroviral therapy electronic patient monitoring system (ART EPMS) was established in 2007 and is the main electronic reporting system for HIV patient care in Namibia. The platform is owned by the Ministry of Health and Social Services (MOHSS) of the Republic of Namibia. The EPMS was based at public sector facilities offering HIV care and the sources of the data are the HIV patient care booklets and registers. Data comprising patient demographics, date of entry into care, laboratory test results, treatment regimen, and appointments are recorded by healthcare workers at the point of care in the HIV care booklets and registers. The data are then transcribed by data clerks into the electronic database, where periodic reports are generated in electronic form, and relayed to regional and national levels for data cleaning, aggregation and analysis. For our study, the closing date for data collection was 20 November 2020.

The study population was HIV-infected adults on antiretroviral therapy in the four regions of northern Namibia. For inclusion in the study, participants had to be women and men of 18 years and older, on antiretroviral therapy, to have had at least two measurements of serum creatinine that were at least three months apart, and attending HIV care clinics in Ohangwena, Omusati, Oshana and Oshikoto regions. Serum creatinine measurements obtained earlier than I January 2017 were not considered. For recruitment, at each hospital a status report of active patients registered for HIV management was generated from the electronic database and exported to Microsoft Excel. Active patients were defined as those who would have received treatment at the site within a period of three months up to the point of data collection; routine appointments are three months apart.

Thereafter, a random sample of patients was selected in Microsoft Excel using the simple random selection function, and the required data extracted from the electronic database for each selected participant according to the data collection tool. The data collection tool was piloted on 50 participants and modified accordingly. Selected participants who did not satisfy the eligibility criteria were excluded. The required sample size to estimate the prevalence with minimum random error was calculated as follows: $95 \%$ confidence interval with a half width of $1 \%$, estimated prevalence from the literature of $7 \%$ [14], therefore the required sample size was approximately 2500 participants using the formula for a single proportion.

The two most recent serum creatinine measurements obtained at least three months apart, date of birth, sex, date of initiation of antiretroviral therapy, current antiretroviral treatment, and most recent HIV viral load results were the variables used in the study. Serum creatinine was measured by the Namibia Institute of Pathology (NIP) at laboratories located at the participating hospitals. The NIP reports standardized creatinine and uses National Institute of Standards and Technology standard reference material $967 \mathrm{a}$ and the liquid chromatography IDMS reference method for calibration. The machines use the Jaffe method.

STATA 16.I was used for data analysis. The CKD-EPI equation was used to calculate the eGFR for the two serum creatinine results per participant [19]. Frequency, relative frequency (\%), and 95\% confidence intervals were used to estimate the prevalence of chronic disease using an eGFR of less than $60 \mathrm{~mL} / \mathrm{min} / 1.73 \mathrm{~m}^{2}$ for at least 3 months as the threshold to diagnose CKD. Age, sex and CKD were also assessed by region. The variables age greater than or equal to 45 years and duration on antiretroviral therapy greater than or equal to 108 months were generated based on the mean age and median duration cut points, respectively. Only four variables were assessed for association with CKD. These were chosen based on clinical importance in the literature. All four variables were assessed at univariable level for association with CKD at the 0.2 level of significance, and those variables which were associated at this level were added to a multiple logistic regression model. The model was assessed using the HosmerLemeshow test and the area under the receiver operating characteristics curve.

\section{Ethics considerations}

The Health Research Ethics Committee (HREC) of the University of Stellenbosch approved the study with a waiver of informed consent (HREC reference number: S20/04/108). The MOHSS of Namibia and the NIP gave approval for the study as required by local regulations.

\section{RESULTS}

A total of I 993 participants were included in this study. The participant flow is shown in Figure I. Women constituted about $68 \%$ of participants, with Omusati region having the lowest proportion of women at 59\% compared to the other regions. The mean age of participants was 44.5 \pm I 1.5 years. Table I shows the distribution of age and sex 
Table I. Characteristics of participants.

\begin{tabular}{|c|c|c|c|c|c|c|c|}
\hline & \multirow[t]{2}{*}{ Number } & \multicolumn{2}{|c|}{ Sex N (\%) } & \multirow[t]{2}{*}{$\begin{array}{c}\text { Age (years) } \\
(\text { mean } \pm S D)\end{array}$} & \multirow{2}{*}{$\begin{array}{c}\text { Duration on } \\
\text { ART (months) } \\
\text { (median) (IQR) }\end{array}$} & \multirow{2}{*}{$\begin{array}{l}\text { Viral load less } \\
\text { than } 1000 \mathrm{cps} / \\
\mathrm{mL} \text {, proportion } \\
(\%)(95 \% \mathrm{Cl})\end{array}$} & \multirow{2}{*}{$\begin{array}{l}\text { TDF use, } \\
\text { proportion (\%) } \\
(95 \% \mathrm{Cl})\end{array}$} \\
\hline & & Female & Male & & & & \\
\hline Overall & 1993 & I $362(68.3)$ & $631(31.7)$ & $44.5 \quad \mid 1.5$ & $107(62-149)$ & 97.3 (96.5-98) & 94.1 (93-95.1) \\
\hline Ohangwena & 245 & | 86 (75.9) & $59(24.1)$ & $44 \quad 12.1$ & $94(46-145)$ & $97.6(94.8-99.1)$ & 96.7 (93.7-98.6) \\
\hline Omusati & 450 & $267(59.3)$ & I 83 (40.7) & $40.8 \quad 11.7$ & $92(34-153)$ & $96.0(93.8-97.6)$ & 97.1 (95. I-98.5) \\
\hline Oshana & 670 & 474 (70.7) & $196(29.3)$ & $46.4 \quad 10.6$ & $118(76-146)$ & $98.8(97.7-100)$ & $89.4(86.8-91.6)$ \\
\hline Oshikoto & 628 & $435(69.3)$ & 193 (30.7) & $45.5 \quad 11.5$ & $105(66-148)$ & $96.7(94.9-97.9)$ & $96.0(94.2-97.4)$ \\
\hline
\end{tabular}

ART, antiretroviral therapy; Cl, confidence interval; cps/mL, copies per millilitre; IQR, interquartile range; N, number; SD, standard deviation; TDF, tenofovir disoproxil fumarate.

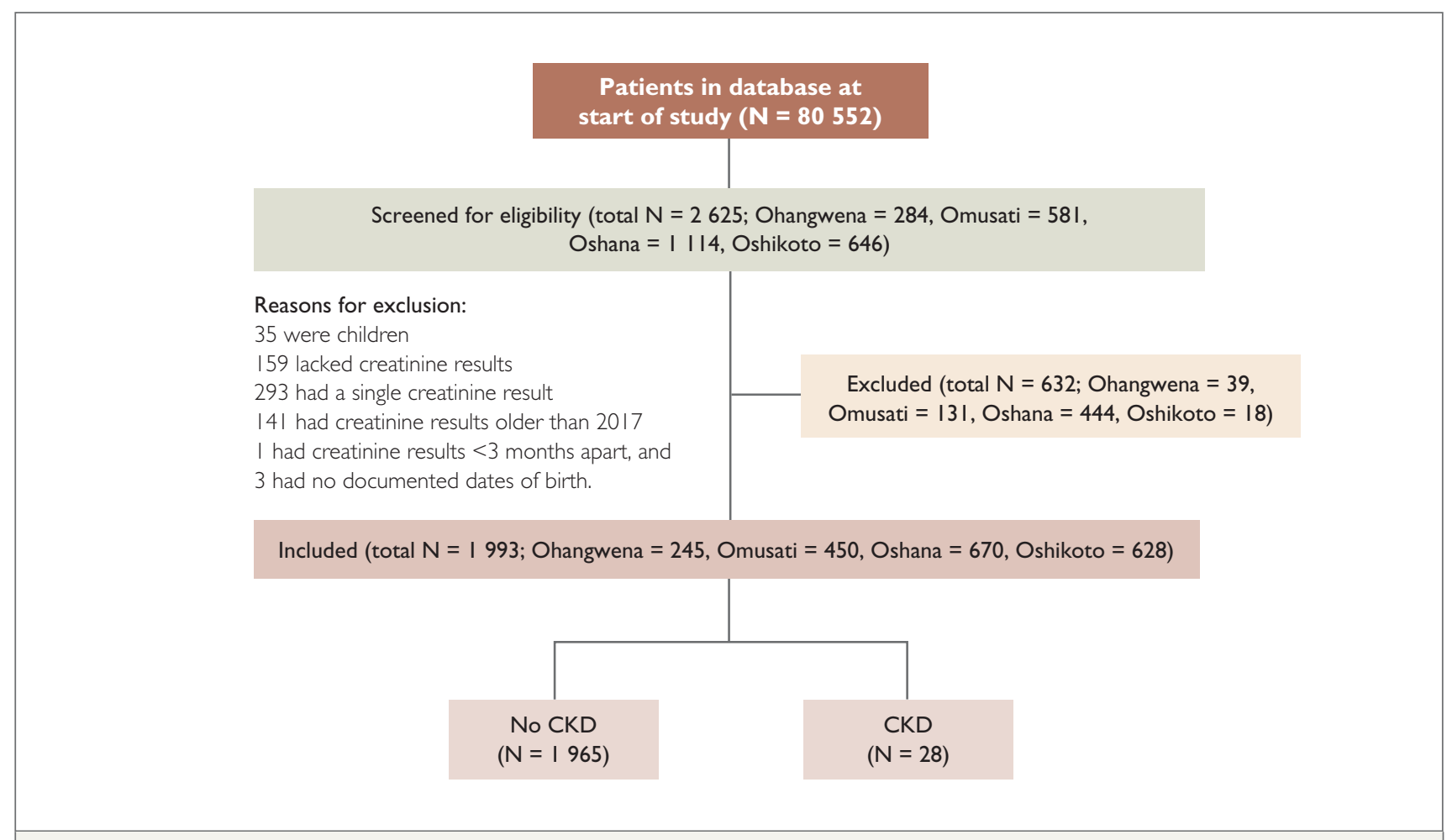

Figure I. Flowchart of participant selection process.

by region. Oshana region had the oldest participants on average compared to the three other regions. The median duration of antiretroviral therapy use was 107 months with an interquartile range of 62 to 149 months.

The proportion of patients who were virally suppressed was 97.0\% (95\% Cl 96.5, 98.0\%), and those who had a history of using antiretroviral therapy including TDF were 94.0\% (95\% Cl 93.0, 95.1\%). There were 28 patients with both eGFR measurements below $60 \mathrm{~mL} / \mathrm{min} / 1.73 \mathrm{~m}^{2}$, yielding a prevalence of CKD of I.4\% (95\% Cl I.0, 2.0\%).
Participants with an initial eGFR less than $60 \mathrm{~mL} / \mathrm{min} /$ $1.73 \mathrm{~m}^{2}$ were $2.6 \%(95 \% \mathrm{Cl} 2.0,3.4 \%)$, while those with a later eGFR less than $60 \mathrm{~mL} / \mathrm{min} / 1.73 \mathrm{~m}^{2}$ were $2.8 \%(95 \%$ Cl 2.1, 3.6\%).

Table 2 shows the prevalence of CKD by region, with the Oshikoto region having the highest occurrence of CKD compared to the other regions, though the values are all quite low. There was no statistically significant difference in prevalence of CKD by region; chi-squared statistic 3.0 and $P$ value 0.4 . 
Table 2. Prevalence of chronic kidney disease.

\begin{tabular}{lcc|} 
& \multicolumn{2}{c}{ CKD, N (total); \% $(95 \% \mathrm{Cl})$} \\
Overall (1993) & $28(1993)$ & $1.4 \%(1.0,2.0)$ \\
Ohangwena (245) & $4(245)$ & $1.6 \%(0.5,4.1)$ \\
Omusati (450) & $3(450)$ & $0.7 \%(0.1,1.9)$ \\
Oshana (670) & $9(670)$ & $1.3 \%(0.6,2.5)$ \\
Oshikoto (628) & $12(628)$ & $1.9 \%(1.0,3.3)$ \\
\hline
\end{tabular}

$\mathrm{Cl}$, confidence interval; CKD, chronic kidney disease; $\mathrm{N}$, number. ated with chronic kidney disease, after adjusting for sex [adjusted odds ratio (AOR) $13.2(95 \%$ Cl 3.I, 56.0)]. Participants with CKD were 13 times more likely to be 45 years or older than those with no CKD. Similarly, male sex was significantly associated with CKD [AOR 3.5 (1.6, 7.7)]. Therefore, patients with CKD were 3.5 times more likely to be male than those without CKD. Viral load and duration of treatment were not significantly associated with chronic kidney disease, after adjusting for age and male sex and were thus excluded from the final model.

Table 3. Univariable analysis for risk factors of chronic kidney disease.

\begin{tabular}{|c|c|c|c|c|c|}
\hline \multirow[t]{2}{*}{ Variable } & & \multicolumn{2}{|c|}{ CKD } & \multirow[t]{2}{*}{$\begin{array}{l}\text { Crude odds ratio } \\
\qquad(95 \% \mathrm{Cl})\end{array}$} & \multirow[t]{2}{*}{$P$ value } \\
\hline & & Present & Absent & & \\
\hline \multirow{2}{*}{ Age $\geq 45$ years } & No & 2 & 1030 & I (reference) & \multirow{2}{*}{$<0.00$ I } \\
\hline & Yes & 26 & 935 & $14.3(3.4,60.5)$ & \\
\hline \multirow{2}{*}{ Male sex } & No & 10 & 1352 & I (reference) & \multirow{2}{*}{$<0.00$ । } \\
\hline & Yes & 18 & 613 & $4(1.8,8.7)$ & \\
\hline \multirow{2}{*}{ Viral load $\geq 1000$ copies $/ \mathrm{mL}$} & No & 27 & 1907 & I (reference) & \multirow{2}{*}{0.848} \\
\hline & Yes & । & 58 & $1.2(0.2,9.1)$ & \\
\hline \multirow{2}{*}{ Treatment duration $\geq 108$ months } & No & 12 & 991 & I (reference) & \multirow{2}{*}{0.426} \\
\hline & Yes & 16 & 974 & $1.4(0.6,2.9)$ & \\
\hline
\end{tabular}

$\mathrm{Cl}$, confidence interval; CKD, chronic kidney disease.

Table 4. Multivariable logistic regression analysis of patient characteristics on occurrence of chronic kidney disease.

\begin{tabular}{|llc|}
\hline Variable & $\begin{array}{l}\text { Adjusted odds } \\
\text { ratio }(95 \% \mathrm{Cl})\end{array}$ & P value \\
Age $\geq 45$ years & I3.2 $(3.1,56)$ & $<0.001$ \\
Male sex & $3.5(1.6,7.8)$ & 0.002 \\
Logistic regression & Chi-squared $=37, \mathrm{P}=<0.001$ \\
Hosmer-Lemeshow test & Chi-squared $=3, \mathrm{P}=0.215$ \\
Area under ROC curve & 0.80 \\
\hline
\end{tabular}

$\mathrm{Cl}$, confidence interval; $\mathrm{ROC}$ receiver operating characteristics.

Table 3 shows the results of the crude analysis of risk factors for CKD. Age equal to or greater than 45 years [crude odds ratio (COR) 14.3 (95\% Cl 3.4, 60.5)] and male sex [COR $4(95 \% \mathrm{Cl}$ I.8, 8.7)] were risk factors. Viral load $\geq 1000$ copies $/ \mathrm{mL}$ and duration of treatment $\geq 108$ months were not associated with CKD.

\section{DISCUSSION}

The prevalence of CKD in this study of HIV-infected adults from northern Namibia was 1.4\%. This is lower than the prevalence reported in systematic reviews on CKD in the HIV-infected population in Africa $[5,14,15,20]$. We found that age $\geq 45$ years and being male were risk factors. Having a viral load $\geq 1000$ copies/mL and longer duration on antiretroviral therapy were not associated with CKD in our study.

The occurrence of CKD in HIV-infected populations in subSaharan Africa varies by country, study, equation used to estimate GFR, and criteria for diagnosing CKD $[14,15$, 21-23]. The prevalence tends to be higher when using a broader definition of CKD that includes albuminuria, eGFR, and structural kidney abnormalities compared to eGFR alone [2I-24]. Many studies do not establish chronicity but rather rely on the exclusion of an acute illness that can be a cause of kidney disease during the period when kidney function is assessed [4,15]. In this study, we established chronicity by using two consecutive estimates of GFR that were at least three months apart. Furthermore, we calcu- 
lated the eGFR using the CKD-EPI equation, as is recommended.

CKD occurrence rises with increasing age, and this has also been observed in HIV-infected populations [ [ 6,21,23]. We observed this association in our study. However, it is important to consider that age may also be a marker of the presence of HIV infection for a longer duration and of other comorbidities that are risk factors for CKD [25]. While being male was a risk factor for CKD in our study, other studies have demonstrated that there is no difference in the occurrence of CKD by sex [26]. However, kidney failure tends to be higher among men compared to women [26]. Establishing virological control through antiretroviral therapy is an important part of measures to reduce the occurrence of kidney disease in HIV, and a good response to antiretroviral therapy improves kidney function $[10,27]$. The proportion of participants in our study with virological control was $97 \%$ and this may explain the low prevalence we are reporting. Additionally, the prevalence of diabetes mellitus in the Namibian population, based on the 2013 Namibia Demographic and Health Survey, was low at $5.1 \%$ [28]. The low prevalence of an important determinant of CKD in the general population may also help explain the low prevalence of CKD we are reporting.

\section{Strengths and limitations}

The strengths of our study are that we carried out a multicentre study with a sample of 1993 participants, used the recommended CKD-EPI equation, and we established chronicity. However, as we relied on existing records, we were not able to collect data on established risk factors for CKD such as diabetes mellitus and hypertension. We used only eGFR to diagnose CKD, and data on albuminuria/ proteinuria and abnormalities of kidney structure were not available. This may imply that we underestimated the prevalence of CKD in this population. Furthermore, we did not assess TDF use as a risk factor for CKD as patients with CKD are deliberately not given TDF-containing antiretroviral therapy, as per our guidelines.

\section{CONCLUSIONS}

The burden of CKD among HIV-infected adults on antiretroviral therapy in northern Namibia was low at 1.4\%. Since older age was established as a risk factor of CKD in this population, we recommend routine assessment of eGFR in older patients with HIV infection.

\section{Supplementary materials}

The characteristics of study participants and the excluded patients are available via the supplementary materials on the African Journal of Nephrology website.

\section{CKD-EPI Equation}

\section{Female}

eGFR $=144 \times($ serum creatinine/61.9) $-0.329 \times(0.993)$

$\wedge$ age if serum creatinine is less than or equal to $61.9 \mathrm{\mu mol} / \mathrm{L}$ [19].

eGFR $=144 \times(\text { serum creatinine/61.9 })^{\wedge}-1.209 \times(0.993)$

$\wedge$ age if serum creatinine is greater than $61.9 \mu \mathrm{mol} / \mathrm{L}$ [19].

Male

eGFR $=|4| \times\left(\right.$ serum creatinine/79.6) ${ }^{\wedge}-0.4|| \times(0.993)$

$\wedge$ age if serum creatinine is less than or equal to $79.6 \mu \mathrm{mol} / \mathrm{L}$ [19].

eGFR $=14 \mid \times\left(\right.$ serum creatinine/79.6) ${ }^{\wedge}-1.209 \times(0.993)$

$\wedge$ age if serum creatinine is greater than $79.6 \mu \mathrm{mol} / \mathrm{L}$ [19].

\section{Declarations}

\section{Consent for publication}

The authors obtained consent to publish from the MOHSS of Namibia.

\section{Availability of data}

The data set analysed during this study is available upon request from the authors.

\section{Funding}

This study was not funded by any specific grant from any funding agency.

\section{Competing interests}

The authors declare that they have no competing interests.

\section{Acknowledgements}

The authors acknowledge the hospital authorities, data clerks and NIP staff for their assistance and cooperation during the data collection process.

\section{REFERENCES}

I. Levin A, Stevens P, Bilous R, Coresh J, De Francisco A, De Jong P. Kidney Disease: Improving Global Outcomes (KDIGO) CKD work group. KDIGO 2012 clinical practice guideline for the evaluation and management of chronic kidney disease. Kidney Int Suppl. 2013; 3: I - I50. doi: I0.1038/kisup.2012.73.

2. Davids MR, Chothia MY. Chronic kidney disease for the primary care clinician. S Afr Fam Prac. 2019; 61:19-24. doi:10.4102/safp. v6li5.494I.

3. Webster AC, Nagler E V., Morton RL, Masson P. Chronic kidney disease. Lancet. 2017; 389:1238-1252. doi:10.1016/S01406736(I 6)32064-5.

4. Davids MR, Benghanem Gharbi M. Global considerations in kidney disease: Africa. In: Yu ASL, Chertow GM, Luyckx VA, Marsden PA, Skorecki K, Taal MW, editors. Brenner \& Rector's The Kidney. IIth ed. Philadelphia, PA: Elsevier; 2019. pp. 2493-25I6.

5. Stanifer JW, Jing B, Tolan S, Helmke N, Mukerjee R, Naicker S, et al. The epidemiology of chronic kidney disease in sub-Saharan Africa: a systematic review and meta-analysis. Lancet Glob Health. 2014; 2:el74-el81. doi: I0.1016/S22 |4-109X(| 4)70002-6.

6. Noubiap JJN. Diabetic nephropathy in Africa: A systematic review. World J Diabetes. 20 I5; 6:759-773. doi:I0.4239/wjd.v6.i5.759. 
7. Davids MR, Marais N, Jacobs JC. South African Renal Registry Annual Report 2015. Afr J Nephrol. 2017; 1:201-213. doi: I0.21804/20-1-2583.

8. Wools-Kaloustian KK, Gupta SK. Will there be an epidemic of HIV-related chronic kidney disease in sub-Saharan Africa? Too soon to tell. Kidney Int. 2008; 74:845-847. doi: I 0. I038/ki.2008.326.

9. Magodoro IM, Esterhuizen TM, Chivese T. A cross-sectional, facility based study of comorbid non-communicable diseases among adults living with HIV infection in Zimbabwe. BMC Res Notes. 2016; 9:379-388. doi: 10.1 | 86/s | 3104-016-2 I 87-z.

10. Swanepoel CR, Atta MG, D'Agati VD, Estrella MM, Fogo AB, Naicker S, et al. Kidney disease in the setting of HIV infection: conclusions from a Kidney Disease: Improving Global Outcomes (KDIGO) Controversies Conference. Kidney Int. 20। 8; 93:545-559. doi: 10.1016/j.kint.20 I7.1 I.007.

I I. Wyatt CM. Kidney disease and HIV Infection. Top Antivir Med. 2017; 25:13-16.

12. Govender K, Suleman F, Moodley Y. Clinical risk factors for in-hospital mortality in older adults with HIV infection: findings from a South African hospital administrative dataset. Pan Afr Med J. 2017; 26: I-II. doi:I0.I I604/pamj.2017.26.126.I1000.

13. Mgori NK, Mash R. HIV and/or AIDS-related deaths and modifiable risk factors: A descriptive study of medical admissions at Oshakati Intermediate Hospital in Northern Namibia. Afr J Prim Health Care Fam Med. 2015; 7:883-889. doi:10.4102/phcfm.v7il.883.

14. Ekrikpo UE, Kengne AP, Bello AK, Effa EE, Noubiap JJ, Salako BL, et al. Chronic kidney disease in the global adult HIV-infected population: A systematic review and meta-analysis. PLoS ONE. 2018; | 3:e0195443. doi: I 0. I 37|/journal.pone.0195443.

15. Kaze AD, Ilori T, Jaar BG, Echouffo-Tcheugui JB. Burden of chronic kidney disease on the African continent: a systematic review and meta-analysis. BMC Nephrol. 20 18; 19:125-135. doi: I0.I 186/ s| 2882-0| 8-0930-5.

16. Kaboré NF, Poda A, Zoungrana J, Da O, Ciaffi L, Semdé A, et al. Chronic kidney disease and HIV in the era of antiretroviral treatment: findings from a 10 -year cohort study in a west African setting. BMC Nephrol. 2019; 20: I55- | 64. doi: I0. I | 86/s | 2882-019-I335-9.

17. Republic of Namibia Ministry of Health and Social Services. Preliminary findings of Namibia Population-Based HIV Impact Assessment (NAMPHIA) 2017. 2018. Available: https://phia.icap. columbia.edu/wp-content/uploads/2018/10/33462•NAMPHIA-SS_ A4_B.v4l.pdf.

18. Republic of Namibia Ministry of Health and Social Services. National guidelines for antiretroviral therapy. https://www.namhivsociety.org/ media/hivsoc/Pdf/art_guidelines/Final\%20ART\%20Guidelines.pdf.

19. Levey AS, Stevens LA, Schmid CH, Zhang YL, Castro AF, Feldman $\mathrm{HI}$, et al. A new equation to estimate glomerular filtration rate. Ann Intern Med. 2009; I 50:604-6I2. doi: I0.7326/0003-48 I9- I50-9200905050-00006.

20. Bikbov B, Purcell CA, Levey AS, Smith M, Abdoli A, Abebe M, et al. Global, regional, and national burden of chronic kidney disease, 1990-2017: a systematic analysis for the Global Burden of Disease Study 2017. Lancet. 2020; 395:709-733. doi:10.1016/S01406736(20)30045-3.

21. Marie Patrice H, Moussa O, Francois K, Yacouba M, Hugo M, Henry L. Prevalence and associated factors of chronic kidney disease among patients infected with human immunodeficiency virus in Cameroon. Iran J Kidney Dis. 2018; 12:268-274.

22. Cailhol J, Nkurunziza B, Izzedine H, Nindagiye E, Munyana L, Baramperanye $\mathrm{E}$, et al. Prevalence of chronic kidney disease among people living with HIV/AIDS in Burundi: a cross-sectional study. BMC Nephrol. 20 I I; 12:40-48. doi: I 0. I | 86/ |47 |-2369- | 2-40.

23. Glaser N, Phiri S, Bruckner T, Nsona D, Tweya H, Ahrenshop N, et al. The prevalence of renal impairment in individuals seeking HIV testing in urban Malawi. BMC Nephrol. 2016; 17:|86-19|.
24. Obirikorang C, Osakunor DNM, Ntaadu B, Adarkwa OK. Renal function in Ghanaian HIV-infected patients on highly active antiretroviral therapy: A case-control study. PLoS ONE. 2014; 9:e99469. doi:I0.137|/journal.pone.0099469.

25. Guaraldi G, Malagoli A, Calcagno A, Mussi C, Celesia BM, Carli F, et al. The increasing burden and complexity of multi-morbidity and polypharmacy in geriatric HIV patients: a cross-sectional study of people aged 65-74 years and more than 75 years. BMC Geriatr. 20।8; | 8:99-108. doi: | 0.1 | 86/s | 2877-0 | 8-0789-0.

26. Goldberg I, Krause I. The role of gender in chronic kidney disease. Eur Med J. 2016; 1:58-64.

27. Fabian J, Naicker S, Goetsch S, Venter WDF. The clinical and histological response of HIV-associated kidney disease to antiretroviral therapy in South Africans. Nephrol Dial Transplant. 20।3; 28: I543- I554. doi: I 0. I093/ndt/gft0 I 0.

28. Adekanmbi VT, Uthman OA, Erqou S, Echouffo-Tcheugui JB, Harhay MN, Harhay MO. Epidemiology of prediabetes and diabetes in Namibia, Africa: A multilevel analysis. J Diabetes. 2019; I I:I61-172. doi: | 0.1 | | |/|753-0407.12829. 